\title{
The Impact of Mobile Devices on Sleep Quality among Subjects of Primary Health Care Settings in Riyadh, Saudi Arabia
}

\author{
Ibrahim Muhaydib Al-Muhaydib", ${ }^{1,2}$, Bader AbdulAziz Al-Tulihi',2, \\ Osamah Saleh Alnafisah ${ }^{1,2}$, Abdulrahman Muhaidib Almuhaidib ${ }^{3}$, \\ Ammar Abdulkareem Al-Sayegh ${ }^{4}$ and Omar Muhaidib Al-Muhaidib ${ }^{3}$
}

\author{
${ }^{1}$ Department of Family Medicine, Ministry of National Guard - Health Affairs, Riyadh, Saudi Arabia. \\ ${ }^{2}$ King Abdullah International Medical Research Center, Riyadh, Saudi Arabia. \\ ${ }^{3}$ College of Medicine - Imam Muhammed Ibn Saud Islamic University, Riyadh, KSA. \\ ${ }^{4}$ Department of Internal Medicine, Prince Mohammed Bin Abdul Aziz Hospital, Riyadh, KSA.
}

$$
\text { http://dx.doi.org/10.13005/bbra/2940 }
$$

(Received: 16 July 2021; accepted: 24 September 2021)

\begin{abstract}
Smartphone use has been related to health problems. Studies have indicated that the use of smartphones is associated with Sleep disturbances. However, little is known about the association between Smartphone addiction and sleep quality in Saudi Arabia. To determine the relationship between sleep quality and the use of a smartphone, specifically before sleep. A cross-sectional study was conducted at four primary healthcare centers with a total of 205 patients participated in the study using a convenience sampling technique. The Pittsburgh Sleep Quality Index (PSQI) and Smartphone Addiction Scale - Short Version (SAS-SV) were used to measure association of Sleep Quality and Smartphone addiction. Two-hundred-fiveparticipants enrolled in the study, of which 99 were female $(51 \%)$ with a response rate of $84 \%$. Mean age was $36 \pm 13$ years for all participants. Mean PSQI scores were higher among females, single, frequent smartphone users, and unemployed sub- categories; thus, they indicated poorer sleep quality. There was also a significant negative association between age and PSQI scores $t(193)=$ $\mathbf{- 2 . 4 5}, p=0.015$. Finally, there was a significant positive association between mean SAS-SV score and categorical global PSQI scores $\mathrm{t}(193)=3.4, \mathrm{p}=0.001$. Our study showsahigh prevalence of smartphone addiction and poor sleep quality. Younger age, being single, heavy usage hours of smartphones, and high SAS-SV are significant factors associated with poorer sleep quality.
\end{abstract}

Keywords: Insomnia; Sleep Quality; Saudi Arabia; Smartphone addiction.

The role of sleep on cognitive, physical and wellbeing of individuals is well established. ${ }^{1}$ Good sleep quality might be related and linked to several factors, including social life aspects, general health status, environmental factors, and stress. Moreover, it is essential for health and life quality in all people. It has been shown, that poor sleep quality can lead to multiple health related issues that might lead to physical or mental disorders. ${ }^{2}$ Thus, studying and identifying possible contributed factors for poor sleep quality is essential.

Over-use of smartphones is one of the environmental factors that might be considered a possible contributor for poor quality of sleeping. The mobile phone has evolved in recent decades 
to become a smartphone and caused a dramatic change in societies worldwide, as well as transforming communication. These tools now provide communication, entertainment, and education functions. ${ }^{3}$ The term of smartphone in English is defined as a "mobile phone that performs many of the functions of a computer, typically having a touchscreen interface, Internet access, and an operating system capable of running downloaded applications". ${ }^{4}$

It was in $1980 \mathrm{~s}$, were phones were started to be enhanced using computer science and technology. Since then, terms as with mobile phones, cell phones, and smart phones were started to be used gradually and interchangeably. ${ }^{5,6}$ Usage of smartphones has substantially increased in recent years in all communities. Kingdom of Saudi Arabia ranked third in the world in the number of smartphone users. According to recent reports, they make up $72.8 \%$ of the population. ${ }^{3}$

A growing attention in medical and academic fields was observed for health hazards related to sleep deprivation and its relationship with over-use of smartphones. ${ }^{1,7,8}$ A cross-sectional study conducted in Japan among adolescents in 2011 showed that there was a strong correlation between smartphone usage after lights tuned off and sleep related issues, as with poor sleep quality, sleep duration, daytime sleepiness, and insomnia among the participants. ${ }^{9}$ Some other studies reported other sleep related issues as with poor sleep efficiency, rapid eye movement sleep, and sleep latency among participants. ${ }^{1,10-13}$

There have been few studies performed in the Middle East. For example, in 2018, a regional study among medical students at King Abdul-Aziz University (KAU), showed a high prevalence for frequent smartphones usage accounting for $73 \%$ of the participants for a duration that exceeds 5 hours per day. Similarly, most of the participants have reported poor sleep quality. The study has concluded that smartphone dependency had been associated with poor sleep quality and latency. ${ }^{14}$

However, the relationship between overuse of smartphones and its effect on sleeping is a matter of debate with no concrete evidence. Therefore, this study was conducted to investigate the relationship between the excessive use of smartphones and quality of sleep in Primary Health Care Centers at the Ministry of National
Guard, Health Affairs in Riyadh, Saudi Arabia. Our secondary objective was determining the factors associated with poor sleep quality from demographic data.

\section{MATERIAL AND METHODS}

\section{Study design and settings}

This study was a descriptive crosssectional study. It was conducted at four primary health care centers at Ministry of National Guard, Health Affairs in Riyadh, Kingdom of Saudi Arabia: National Guard Comprehensive Specialized Clinic (NGCSC), Health Care Specialty Center (HCSC), King Abdulaziz City Housing (Iskan-Yarmouk) and King Saud City Housing (Dirab).

\section{Study population}

This study targeted all adults 17 years old or older, including both genders, who were followed in four previous NGHA centers in Riyadh. A patient diagnosed with a preexisting sleeping disorder, mental disorder (e.g., depression or insomnia), non-smartphone users, children and pregnant females were excluded from this study.

\section{Sampling technique}

A sample size frame was unavailable for the four targeted centers; thus, a convenience sampling technique was used to distribute the selfadministered questionnaire among the participants. As we used such a technique, the sample in our study is unlikely to represent the overall population, and sampling size calculations were not needed.

\section{Data collection methods, instruments, and measurements}

The participants completed a selfadministered questionnaire, which includes questions that were taken from the Pittsburgh Sleep Quality Index (PSQI) and Smartphone Addiction Scale - Short Version (SAS-SV).

In addition, questions about demographic and personal/professional characteristics were added as in gender, age, marital status, working time, usage hours for smartphones, and four other questions to meet the excluding criteria as with pregnancy, being diagnosed with mental or sleeping disorders and type of phone being used. The questionnaire was initially developed in English then was translated to Arabic ${ }^{15}$. The questionnaire was adapted after the comprehensive literature search, and the content was validated by 
Kwon et al. in $2013{ }^{16}$ and Buysse et al. (1988) ${ }^{17}$. The validity of translation was ensured by forwardbackward validation methods. The questionnaire pre-testing was conducted on ten subjects from our target population to assure clarity and accuracy of the tool. The approximate time to complete the questionnaire was 4 to $5 \mathrm{~min}$. The targeted subjects were asked to complete the questionnaire after obtaining their consent.

Two scales were adopted in our study, PSQI and SAS-SV. PSQI is a ten-item scale that aims to measure seven components that affect sleep quality. It was developed by Buysse et al. (1988) ${ }^{17}$. and was validated with high internal consistency and a Cronbach's alpha of $0.83{ }^{18,19}$. Each of the seven components will have a grade between 0 (which indicates no sleep difficulties) and 3 (which indicates severe difficulty within the component). The higher the overall score for all seven components, the higher the sleep issues for the participants, with an overall score ranging between 0 to 21. A cut-off number of 5 was selected where an overall mean response for a participant of 5 or more will indicate that he most likely has a sleep difficulty 17,20 .

The SAS-SV is a ten-item score that was developed to measure the addiction of smartphone usage. It was developed by Kwon et al. in $2013^{16}$. The SAS-SV has a Cronbach's alpha correlation coefficient of 0.91 , which is considered within the desired range ${ }^{21}$. For each item or phrase, the respondent will choose among six answers of a
Likert scale ranging from strongly disagree to strongly agree, with an overall score ranging from 10 to 60 , where higher scores indicate higher smartphone addiction. A cut-off mean score of 31 for males and 33 for females was selected to indicate smartphone addictions based on the Kwon et al. validation study ${ }^{16}$.

\section{Data management and analysis plan}

Data were initially managed and coded using Excel, in which exclusion criteria were performed. Then, data were entered and analyzed using a statistical package for the social sciences version 20 (SPSS, 20) software. Categorical variables were reported as frequencies and percentages, while numerical variables were reported as mean and standard deviation. Further analysis was carried out using inferential statistics, using the chi-square test to assess differences between categorical variables and independent student t-test, and ANOVA for numerical variables. Statistical significance was set to be $<0.05$. Scatterplot graphs were used to illustrate the association between study scales, and boxplots were used to illustrate categorical-numerical variables relationships.

\section{Ethical Considerations}

The study was conducted after getting approval from the Institutional Research Board (IRB) of King Abdullah International Medical Research Center KAIMRC. All data, both hard and soft copies, were stored within MNGHA premises and access by the research team only.

Table 1. Baseline characteristics and their mean PSQI score for sleep quality

\begin{tabular}{|c|c|c|c|c|c|c|c|}
\hline Variable & $\begin{array}{l}\text { Sub- } \\
\text { category }\end{array}$ & $\begin{array}{c}\text { Count/ } \\
\text { Mean } \pm \text { S.D. }\end{array}$ & Percentage & $\begin{array}{c}\text { Mean } \\
\text { SAS_SV } \\
\text { score } \\
\text { (out of } 60 \text { ) }\end{array}$ & S.D. & $\begin{array}{c}\text { Mean } \\
\text { PSQI } \\
\text { Score } \\
\text { (out of } 21 \text { ) }\end{array}$ & S.D. \\
\hline Age & & $36 \pm 13$ & - & - & - & - & - \\
\hline \multirow[t]{2}{*}{ Gender } & Male & 96 & $49 \%$ & 33 & 12 & 6 & 3 \\
\hline & Female & 99 & $51 \%$ & 33 & 11 & 7 & 3 \\
\hline \multirow[t]{2}{*}{ Social Status } & Single & 72 & $37 \%$ & 36 & 9 & 7 & 2 \\
\hline & Ever Married & 123 & $63 \%$ & 32 & 12 & 6 & 3 \\
\hline Usage Hours & 1-4 Hours & 72 & $37 \%$ & 28 & 11 & 6 & 3 \\
\hline \multirow[t]{2}{*}{ of Smartphones } & 5-6 Hours & 70 & $36 \%$ & 35 & 10 & 6 & 2 \\
\hline & $>6$ Hours & 53 & $27 \%$ & 38 & 10 & 7 & 3 \\
\hline \multirow[t]{3}{*}{ Worktime } & Morning Only & 100 & $51 \%$ & 33 & 10 & 6 & 3 \\
\hline & Morning/Night & 26 & $13 \%$ & 35 & 14 & 6 & 3 \\
\hline & Not working & 69 & $35 \%$ & 33 & 11 & 7 & 3 \\
\hline
\end{tabular}


All data were anonymous, kept confidential, and used for research purposes only. A consent form was obtained from each participant before they completed the questionnaire.

\section{RESULTS}

A total of 205 had participated in our study, of which 99 were female $(51 \%)$ with a response rate of $84 \%$. For social status, small numbers were noticed for the divorced category (five, 2\%); thus, they were merged into the "Ever married" category, which accounts for most of the participants $(123,63 \%)$. Smartphone usage was diverse, with a comparable number for participants who were using their smartphones frequently, moderately, or infrequently: $53(27 \%), 70(36 \%)$, and $72(37 \%)$. Most of our participants were doing their jobs in the morning $(100,51 \%)$, while only 26 $(13 \%)$ had also occasionally been working at night. Table 1 gives the baseline characteristics for our participants and their mean PSQI score for sleep quality. Mean PSQI scores were higher among females, single, frequent smartphone users, and unemployed sub-categories; thus, they indicated poorer sleep quality. Figure 1 further illustrates this by comparing different variables and their sub-categories with mean global PSQI scores for sleep quality.

Further analysis of respondent's answers for PSQI scores can be found in Table 2. For subjective sleep quality, most respondents had fairly good to very good subjective sleep quality $(80 \%)$. Similarly, most of the respondents had a good sleep duration $>6 \mathrm{~h}(70 \%)$ and sleep efficiency (69\%). However, most of our respondents reported minimal to fair disturbances during their sleep $(75 \%, 18 \%)$, respectively. More than $90 \%$ of the participants did not use any sleeping medication during the past month, and only $14 \%$ have reported that they have fair or severe daytime dysfunction.

The student's t-test was used to determine the association between mean global PSQI scores with age and SAS-SV scores, while the Chi-square test was used to compare between categorical global PSQI scores using a cut-off point of 5 out of 21 with categorical variables such as gender and social status.

As shown in Table 3, there was a significant negative association between age and PSQI scores $\mathrm{t}(193)=-2.45, \mathrm{p}=0.015$. This means younger participants have significantly poorer sleep quality and higher PSQI scores. Gender and work time period subcategories did not have a statistical
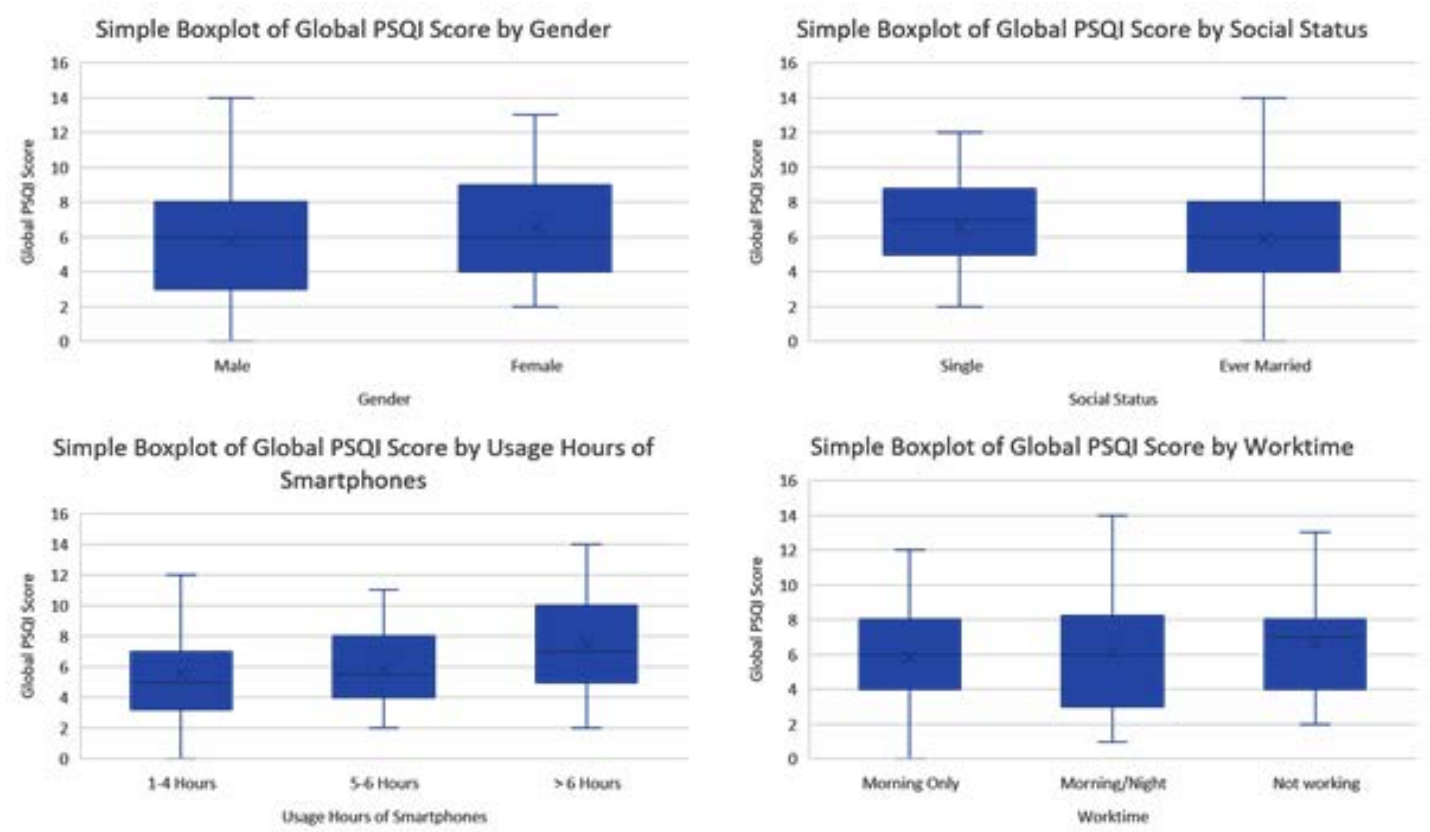

Fig. 1. Boxplots illustrating and comparing gender, social status, usage hours for smartphones, and work time by their sub-categories with mean global PSQI scores among the participants 
significant difference, $\left(\times^{2}(1)=0.58, p=0.45\right)$, $\left(\times^{2}(2)=1.6, p=0.45\right)$, respectively. However, social status and smartphone usage hours of were significantly associated with PSQI, $\left(\times^{2}(1)=4.8, p\right.$ $=0.03),\left(\times^{2}(2)=7.9, p=0.02\right)$, respectively. The single sub-category and frequent smartphone users had higher numbers with abnormal sleep scores

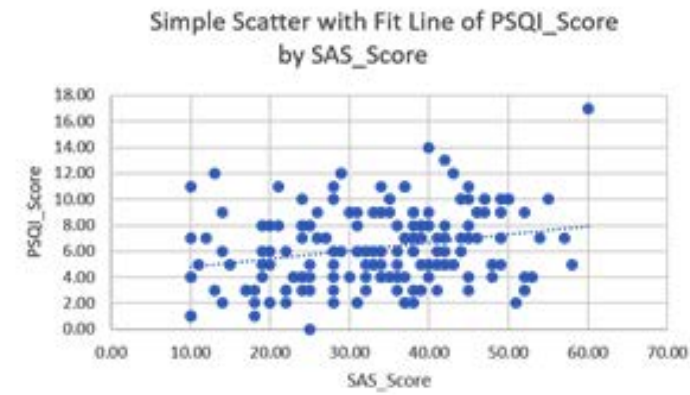

(Table 3), indicating significantly poorer sleep quality among single and frequent smartphone users. Finally, there was a significant positive association between mean SAS-SV score and categorical global PSQI scores using cut off point of 5 out of $21 \mathrm{t}(193)=3.4, \mathrm{p}=0.001$. This means higher SAS-SV scores, which indicates smartphone

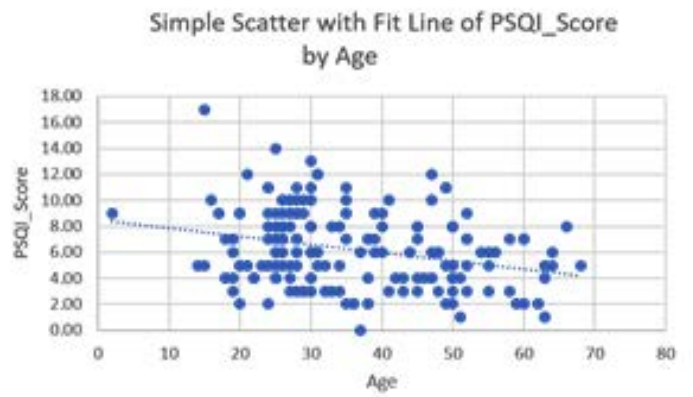

Fig. 2. The linear relationships between PSQI scores and age and SAS-SV scores. The dots were scattered moderately indicating intermediate correlation.

Table 2. PSQI component scores results

\begin{tabular}{|c|c|c|c|}
\hline & & Count & Column N \% \\
\hline \multirow{4}{*}{ Subjective sleep quality } & Very good & 78 & $38.4 \%$ \\
\hline & Fairly good & 86 & $42.4 \%$ \\
\hline & Fairly bad & 34 & $16.7 \%$ \\
\hline & Very bad & 5 & $2.5 \%$ \\
\hline \multirow[t]{4}{*}{ Sleep latency } & No Difficulties & 48 & $23.6 \%$ \\
\hline & Minimal Difficulties & 76 & $37.4 \%$ \\
\hline & Fair Difficulties & 41 & $20.2 \%$ \\
\hline & Severe Difficulties & 38 & $18.7 \%$ \\
\hline \multirow[t]{4}{*}{ Sleep duration } & $>7$ hours & 75 & $36.9 \%$ \\
\hline & 6-7 hours & 67 & $33.0 \%$ \\
\hline & 5-6 hours & 54 & $26.6 \%$ \\
\hline & $<5$ hours & 7 & $3.4 \%$ \\
\hline \multirow[t]{4}{*}{ Sleep efficiency } & $>85 \%$ & 104 & $51.2 \%$ \\
\hline & $75-84 \%$ & 37 & $18.2 \%$ \\
\hline & $65-74 \%$ & 38 & $18.7 \%$ \\
\hline & $<65 \%$ & 24 & $11.8 \%$ \\
\hline \multirow[t]{4}{*}{ Sleep disturbance } & None & 11 & $5.4 \%$ \\
\hline & Minimal & 152 & $74.9 \%$ \\
\hline & Fair & 37 & $18.2 \%$ \\
\hline & Severe & 3 & $1.5 \%$ \\
\hline \multirow[t]{4}{*}{ Use of sleep medication } & Not during the past month & 183 & $90.1 \%$ \\
\hline & Less than once a week & 8 & $3.9 \%$ \\
\hline & Once or twice a week & 10 & $4.9 \%$ \\
\hline & Three or more times a week & 2 & $1.0 \%$ \\
\hline \multirow[t]{4}{*}{ Daytime dysfunction } & No Difficulties & 60 & $29.6 \%$ \\
\hline & Minimal Difficulties & 115 & $56.7 \%$ \\
\hline & Fair Difficulties & 20 & $9.9 \%$ \\
\hline & Severe Difficulties & 8 & $3.9 \%$ \\
\hline
\end{tabular}


Table 3. Association between poor sleep quality, measured by PSQI score and demographic variables

\begin{tabular}{|c|c|c|c|c|c|c|}
\hline \multirow[t]{3}{*}{ Variable } & \multirow[t]{3}{*}{ Sub-category } & \multicolumn{4}{|c|}{ Global PSQI Score } & \multirow[b]{3}{*}{ P-value } \\
\hline & & \multicolumn{2}{|c|}{ Abnormal Sleep Score } & \multicolumn{2}{|c|}{ Normal Sleep scores } & \\
\hline & & $\begin{array}{l}\text { Count/ } \\
\text { Mean } \pm \text { S.D. }\end{array}$ & $\%$ & $\begin{array}{l}\text { Count/ } \\
\text { Mean } \pm \text { S.D. }\end{array}$ & $\%$ & \\
\hline Age $¥$ & & $34 \pm 12$ & - & $39 \pm 14$ & - & $0.02 *$ \\
\hline \multirow[t]{2}{*}{ Gender } & Male & 63 & $65.6 \%$ & 33 & $34.4 \%$ & 0.45 \\
\hline & Female & 70 & $70.7 \%$ & 29 & $29.3 \%$ & \\
\hline \multirow[t]{2}{*}{ Social Status } & Single & 56 & $77.8 \%$ & 16 & $22.2 \%$ & $0.03 *$ \\
\hline & Ever Married & 77 & $62.6 \%$ & 46 & $37.4 \%$ & \\
\hline Usage Hours of & 1-4 Hours & 43 & $59.7 \%$ & 29 & $40.3 \%$ & $0.02 *$ \\
\hline \multirow[t]{2}{*}{ Smartphones } & 5-6 Hours & 46 & $65.7 \%$ & 24 & $34.3 \%$ & \\
\hline & $>6$ Hours & 44 & $83.0 \%$ & 9 & $17.0 \%$ & \\
\hline \multirow[t]{3}{*}{ Worktime } & Morning Only & 65 & $65.0 \%$ & 35 & $35.0 \%$ & 0.45 \\
\hline & Morning/Night & 17 & $65.4 \%$ & 9 & $34.6 \%$ & \\
\hline & Not working & 51 & $73.9 \%$ & 18 & $26.1 \%$ & \\
\hline Smartphone & Addicted & 93 & $76.2 \%$ & 29 & $23.8 \%$ & $0.001 *$ \\
\hline Addiction $¥$ & Non-addicted & 40 & $54.8 \%$ & 33 & $45.2 \%$ & \\
\hline
\end{tabular}

$¥$ Independent t-test was used; Else Chi-square test was used.

* Significant at the 0.05 level.

addiction users had higher PSQI scores indicated poorer sleep quality. The association between PSQI scores with age and SAS-SV scores were further illustrated in Figure 2.

\section{DISCUSSION}

In our study, $63 \%$ of participants had an above cut-off score for SAS-SV, which indicated that they have problematic smartphone usage, which can be a risk for addiction. This result was much higher than the reported percentage of smartphone addiction in the literature, where it was ranged between $27.2 \%$ and $36.5 \%$. ${ }^{3,5}$

This can be explained by the variation in the cut-off number being used in some studies and the current global increase in phone usage due to the COVID-19 pandemic and quarantine in Saudi Arabia.

For sleep difficulties, most of the participants (68\%) in our study had sleep difficulties with a mean PSQI score of $6 \pm 3$. The extent of sleep difficulties among our participants seems to be higher than reported in previous studies. In a study conducted by Amra et al. (2017) ${ }^{1}, 56.1 \%$ of girls and $38.9 \%$ of boys reported poor quality sleep, and late-night cell phone use was associated with poorer sleep quality, which might be comparable to our findings for poorer sleep quality among heavy smartphones users $>6 \mathrm{~h}$ per day. In another study conducted by Mohammadbeigi et al. (2016), using the Cell-Phone Over-Use Scale (COS) and (PSQI) sleep quality score, the prevalence of poor sleep quality was $61.7 \%$ (CI $0.95 ; 57.1 \%, 66.3 \%$ ). ${ }^{2}$

We also found a significant association between poor sleep quality and being younger, single, a heavy phone user, and a high SAS-SV score. This was not reported in previous local studies and can be considered an important factor if a larger random study to be conducted or to be used in linear regression. Despite the promising results we showed, our research has several limitations. We had concerns about our sampling technique and low reported numbers for divorced population or infrequent smartphone users (less than $2 \mathrm{~h}$ per day), which we tried to overcome by increasing the number of participants and by merging divorced and infrequent smartphone users' groups. The COVID pandemic year might be a cause of the relatively low response rate in our study.

Overall, our findings will likely be helpful for initiating a clinical trial to compare sleep quality and its relationship with multiple aspects of smartphone usage. We encourage further studies, given that the number of smartphone users continues to increase. 


\section{Limitations}

We had concerns about our sampling technique and the low reported numbers for divorced population or infrequent smartphone users (less than $2 \mathrm{~h}$ per day), which we tried to overcome by increasing the number of participants and by merging divorced and infrequent smartphone users' groups. The COVID pandemic might account for the relatively low response rate in our study.

\section{CONCLUSION}

Our study showed a high prevalence of smartphone addiction and poor sleep quality for the participants. Younger age, being single, and heavy usage hours of smartphones seem to be indicators for poorer sleep quality. A high SAS-SV score was also found to be a significant indicator of poorer sleep quality.

\section{ACKNOWLEDGEMENTS}

The author would like to thank department of Family Medicine at King AbdulAziz Medical City - National Guard Health Affairs, is highly appreciated for cooperative the PHC centers. The author is also profoundly grateful to the King Abdullah International Medical Research Center (KAIMRC).

\section{Conflict of interest}

We have no Conflict of interest for our article.

\section{Funding Sources}

This research received no funds.

\section{REFERENCES}

1. Amra B, Shahsavari A, Shayan-Moghadam R, et al. The association of sleep and late-night cell phone use among adolescents. Jornal de pediatria. 2017;93(6):560-567.

2. Mohammadbeigi A, Absari R, Valizadeh F, et al. Sleep quality in medical students; the impact of over-use of mobile cellphone and social networks. Journal of research in health sciences. 2016;16(1):46.

3. Alhazmi AA, Alzahrani SH, Baig M, Salawati EM. Prevalence and factors associated with smartphone addiction among medical students at King Abdulaziz University, Jeddah. Pakistan journal of medical sciences. 2018;34(4):984.

4. Alosaimi FD, Alyahya H, Alshahwan H, Al
Mahyijari N, Shaik SA. Smartphone addiction among university students in Riyadh, Saudi Arabia. Saudi Med J. 2016;37(6):675-83. doi:10.15537/Smj.2016.6.14430

5. Alosaimi FD, Alyahya H, Alshahwan H, Al Mahyijari N, Shaik SA. Smartphone addiction among university students in Riyadh, Saudi Arabia. Saudi medical journal. 2016;37(6):675.

6. Srivastava L. Mobile phones and the evolution of social behaviour. Behaviour \& information technology. 2005;24(2):111-129.

7. Eyvazlou M, Zarei E, Rahimi A, Abazari M. Association between overuse of mobile phones on quality of sleep and general health among occupational health and safety students. Chronobiology international. 2016;33(3):293300.

8. Grandner MA. Sleep, Health, and Society. Sleep Med Clin. 2017;12(1):1-22. doi:10.1016/j. jsmc.2016.10.012

9. Munezawa T, Kaneita Y, Osaki Y, et al. The association between use of mobile phones after lights out and sleep disturbances among Japanese adolescents: a nationwide cross-sectional survey. Sleep. 2011;34(8):1013-1020.

10. King DL, Gradisar M, Drummond A, et al. The impact of prolonged violent video gaming on adolescent sleep: an experimental study. Journal of sleep research. 2013;22(2):137-143.

11. Demirci K, Akgonul M, Akpinar A. Relationship of smartphone use severity with sleep quality, depression, and anxiety in university students. J Behav Addict. 2015;4(2):85-92. doi:10.1556/2006.4.2015.010

12. Alshobaili FA, AlYousefi NA. The effect of smartphone usage at bedtime on sleep quality among Saudi non- medical staff at King Saud University Medical City. J Family Med Prim Care. 2019;8(6):1953-1957. doi:10.4103/jfmpc. jfmpc_269_19

13. Rafique N, $\bar{A}$ l-Asoom LI, Alsunni AA, Saudagar FN, Almulhim L, Alkaltham G. Effects of mobile use on subjective sleep quality. Nature and Science of Sleep. 2020;12:357.

14. Ibrahim NK, Baharoon BS, Banjar WF, et al. Mobile phone addiction and its relationship to sleep quality and academic achievement of medical students at King Abdulaziz University, Jeddah, Saudi Arabia. Journal of research in health sciences. 2018;18(3): 00420.

15. Suleiman KH, Yates BC, Berger AM, Pozehl B, Meza J. Translating the Pittsburgh sleep quality index into Arabic. Western Journal of Nursing Research. 2010;32(2):250-268.

16. Kwon M, Kim D-J, Cho H, Yang S. The smartphone addiction scale: development and 
validation of a short version for adolescents. PloS one. 2013;8(12): 833558.

17. Buysse DJ, Reynolds III CF, Monk TH, Berman SR, Kupfer DJ. The Pittsburgh Sleep Quality Index: a new instrument for psychiatric practice and research. Psychiatry research. 1989;28(2):193-213.

18. Salahuddin M, Maru TT, Kumalo A, PandiPerumal SR, Bahammam AS, Manzar MD. Validation of the Pittsburgh sleep quality index in community dwelling Ethiopian adults. Health and quality of life outcomes. 2017;15(1):1-7.

19. Aloba OO, Adewuya AO, Ola BA, Mapayi BM. Validity of the Pittsburgh sleep quality index
(PSQI) among Nigerian university students. Sleep medicine. 2007;8(3):266-270.

20. He J-w, Tu Z-h, Xiao L, Su T, Tang Y-x. Effect of restricting bedtime mobile phone use on sleep, arousal, mood, and working memory: a randomized pilot trial. PloS one. 2020;15(2):e0228756.

21. Sfendla A, Laita M, Nejjar B, Souirti Z, Touhami AAO, Senhaji M. Reliability of the Arabic smartphone addiction scale and smartphone addiction scale-short version in two different Moroccan samples. Cyberpsychology, Behavior, and Social Networking. 2018;21(5):325-332. 